\title{
Observations on progesterone production and clearance in normal pregnant and fetectomized rhesus monkeys (Macaca mulatta)
}

\author{
Rosemarie B. Thau, J. T. Lanman and A. Brinson \\ Center for Biomedical Research, The Population Council, The Rockefeller University, \\ New York, New York 10021, U.S.A.
}

\begin{abstract}
Summary. The metabolic clearance rate (MCR) and production rate (PR) of progesterone were measured in rhesus monkeys both before and during intravenous infusion of progesterone at rates which approximately doubled or tripled the peripheral plasma levels. The monkeys were normally pregnant or fetectomized and were studied during the second half of pregnancy. Raising the peripheral plasma levels did not significantly alter the MCR or the PR of progesterone. We conclude that peripheral progesterone levels are not the factor which controls the PR of progesterone in rhesus monkeys.
\end{abstract}

\section{Introduction}

Progesterone levels and trends during pregnancy vary widely amongst mammals, even those of related species. But for a given species, the levels and trends during pregnancy are characteristic, and the normal range of variability is relatively small. In the rhesus monkey (Macaca mulatta), after some characteristic fluctuations in the first 2 months of pregnancy, peripheral plasma levels in individual animals remain relatively constant and usually in the range of $2-4 \mathrm{ng} / \mathrm{ml}$ up to the time of parturition (Neill, Johansson \& Knobil, 1969). There is no pre-partum decline in peripheral plasma progesterone levels. The progesterone is secreted by the placenta and the ovary; the adrenal is an additional but not significant source (Resko, 1971). In the earliest stages of pregnancy, the ovary is the sole significant site of production. After establishment of placental progesterone production, the individual contributions of the ovary and placenta to the total are difficult to measure and have not been determined, although approximate estimates can be made from progesterone concentrations in uterine and utero-ovarian veins (Walsh, Wolf \& Meyer, 1974). The fetus does not play a direct role in the regulation of progesterone production during pregnancy (Thau, Lanman \& Brinson, 1976a). However, after fetectomy the source of progesterone appears to change from placenta to ovary and a feedback control system is in operation whereby the placenta stimulates the corpus luteum if progesterone production by the placenta becomes inadequate (Lanman, Thau, Sundaram, Brinson \& Bonk, 1975).

Thau et al. (1976b) have reported that, although peripheral progesterone levels in the macaque are stable during the second half of pregnancy, uterine vein levels decline significantly. No sufficient increase in metabolic clearance rate (MCR) nor sufficient decrease in production rate (PR) of progesterone was observed during this period to account for that decline (Thau et al., 1977) and we suggested that increased uterine blood flow was responsible for the declining uterine vein levels of progesterone.

In the present study we have investigated whether the stability of the progesterone production and of the peripheral plasma levels in rhesus monkeys reflected feedback control of progesterone production by peripheral progesterone levels. 


\section{Materials and Methods}

Female rhesus monkeys were housed in individual cages in air-conditioned quarters with a photoperiod of $14 \mathrm{~h} /$ day. The animals were provided with water ad libitum and fed a commercial diet supplemented with fresh fruit. The monkeys were paired with males for Days 11 to 13 of the cycle. Mean cycle length is $26.9 \pm 0.6$ (s.e.m.) days. The first mating day was counted as Day 1 of gestation. Normal pregnancy length in our colony is $164 \pm 7$ (s.d.) days. The pregnancies of all monkeys used in this study were terminated by Caesarean section on various days between Days 153 and 160.

Monkeys 1-7 were studied in normal pregnancy at the times indicated in Table 1 . The fetuses of Monkeys 8, 9 and 10 were removed at 73, 76 and 115 days of gestation respectively and the placentas were left in situ (Lanman et al., 1975). The operations were performed under general anaesthesia between 09:00 and 10:00 h. Monkeys 8-10 were studied before and/or after fetectomy at the times indicated in Table 2. Plasma progesterone concentrations, MCRs and PRs were determined.

\section{$M C R$ and $P R$}

The determination of the MCR was made by the constant infusion method (Tait, 1963). The unanaesthetized animal was restrained in a primate chair. One polyethylene catheter for administration of $\left[1,2,6,7-(\mathrm{N})-{ }^{3} \mathrm{H}\right]$ progesterone (sp. act. $96 \mathrm{Ci} / \mathrm{mmol}$ : New England Nuclear, Boston, Mass.) was threaded into the saphenous vein until the tip lay above the inguinal ligament. This location assures rapid mixing of the infused radioisotope and constant specific activity of the progesterone in the circulation within $20 \mathrm{~min}$ (Thau et al., 1977). A second catheter, inserted into the saphenous vein of the other leg, was used to collect blood samples. The infusion period for $\left[{ }^{3} \mathrm{H}\right.$ ]progesterone was $90 \mathrm{~min}$. Blood samples were collected immediately before and 70,80 and 90 min after the beginning of the infusion. The PR of progesterone was calculated as the product of the MCR and the mean peripheral progesterone concentrations obtained from the 70,80 and 90 min blood samples.

In the present study, two determinations of MCR ('double' MCR), PR and plasma progesterone were made within a 5 -h period: $\left[{ }^{3} \mathrm{H}\right]$ progesterone was infused for 90 min (Test 1 ) followed immediately by the constant infusion for $2 \mathrm{~h}$ of non-radioactive hormone and for an additional $90 \mathrm{~min}$ by the infusion of non-radioactive + labelled hormone (Test 2). Non-radioactive hormone was infused at a rate $(1.2 \mathrm{mg}$ progesterone/day/animal) designed to double or triple the peripheral progesterone levels. Progesterone was infused at higher rates in some animals: $2.4 \mathrm{mg}$ progesterone/day in Monkeys 5, 6 and 9 (68-74 days pregnant), $4.8 \mathrm{mg} / \mathrm{day}$ in Monkey 8 (70 days pregnant) and $12.0 \mathrm{mg} /$ day in Monkey 7 (152 days pregnant). In Monkey 10 the second MCR (Test 2) was carried out after the constant infusion of non-radioactive hormone for $24 \mathrm{~h}$ at the rate of $1.2 \mathrm{mg} / \mathrm{day}$. The accuracy of the infusion pump allowed a constant infusion within a $2 \%$ limit of variation.

Plasma samples were stored at $-20^{\circ} \mathrm{C}$ until analysis. Extraction, purification and determination of the specific radioactivity of $\left[{ }^{3} \mathrm{H}\right]$ progesterone were performed as previously described (Thau \& Lanman, 1975). The mean value of the ${ }^{3} \mathrm{H}$ radioactivity of the 70,80 and $90 \mathrm{~min}$ samples, after correction for procedural losses, was used to calculate the MCRs. The coefficient of variation of these three determinations was below $10 \%$.

The PR determined during non-radioactive progesterone infusion reflected both endogenous production (Test 2) and the contribution from the infused progesterone. From this total value, the contribution from the infused progesterone (usually $1.2 \mathrm{mg} /$ day/animal) was subtracted; the values reported for PR Test 2 represent only endogenous production (Table 1 and 2).

\section{Radioimmunoassays}

Progesterone concentrations were measured by radioimmunoassay (Thorneycroft \& Stone, 1972). The antiserum was produced in rabbits, using $11 \alpha$-succinylhydroxy progesterone-BSA. 
Cross-reactivities were $0.7,0.9$ and $0.2 \%$ for $20 \alpha$-hydroxypregn-4-en-3-one, $17 \alpha$-hydroxyprogesterone and 3 $\beta$-hydroxypregn-5-en-20-one, respectively. The limit of sensitivity of the assay was $25 \mathrm{pg}$. The standard curve ranged from 50 to $800 \mathrm{pg}$. Intra- and inter-assay coefficients of variation were 7.6 and $11.5 \%$, respectively. The coefficient of variation of plasma progesterone concentrations measured at $0,70,80$ and 90 min after the beginning of the infusion was below $8 \%$ for MCR 1 and MCR 2.

The data were analysed statistically by Student's $t$ test and paired $t$ tests.

\section{Normal pregnancy}

\section{Results}

'Double' MCR, PR and plasma progesterone determinations were made in 6 animals (Monkeys 1-6) at 68-75 days, in 3 animals (Monkeys 1-3) at 107-109 days and in 4 animals (Monkeys 1-3 and 7) at 150-152 days of pregnancy (Table 1). The values for the MCR 2 tests were not significantly different from those of MCR $1(P>0.20, P>0.05$ and $P>0.20$ at $68-$ 75, 107-109 and 150-152 days of gestation, respectively; paired $t$ test). The mean value of the MCR 1 tests declined significantly from Day 73 to Day 108, and then increased nearly to its previous values by Day 151 . The mean PR 1 values did not change significantly throughout the same time period. Peripheral plasma progesterone concentrations were normal and ranged from 1.9 to $4.9 \mathrm{ng} / \mathrm{ml}$ before infusion of the non-radioactive progesterone. Within-animal variations as pregnancy progessed were relatively small (see Table 1 ).

Infusions of non-radioactive progesterone for $3 \frac{1}{2} \mathrm{~h}$ at the rate of $1.2 \mathrm{mg} / \mathrm{day}$ raised the peripheral plasma levels by 81 to $242 \%$, but did not significantly reduce the PR; in several monkeys the PR 2 value was higher than the PR 1 value. Infusion of non-radioactive progesterone at rates of 2.4 and $12.0 \mathrm{mg}$ /day raised the peripheral plasma levels by 150 to 441\%. Again, no significant reduction in PR 2 over PR 1 value was observed, except for Monkey 7.

\section{Fetectomized monkeys}

The results for Monkeys 8 and 9 before and after fetectomy are shown in Table 2. Fetectomy did not significantly change the MCR 1, PR 1, PR 2 or progesterone values at 106-109 days of gestation as compared with the values in the same animals measured approximately 5 weeks earlier, before fetectomy. Infusion of non-radioactive progesterone raised peripheral plasma levels by $96-430 \%$ but PR was not affected. The values for MCR 2 tests differed from those of the corresponding MCR 1 by no more than $12.5 \%$. The MCR 2 value was slightly higher $(P<$ $0.05)$ after fetectomy.

Comparison of data for individual fetectomized and normally pregnant animals shows that in 14 out of 18 tests the MCR 2 value was essentially unchanged from the MCR 1 value (the difference was below $20 \%$ after the infusion of non-radioactive progesterone).

\section{Discussion}

Increases in the peripheral plasma level of progesterone induced by progesterone infusion for $3 \frac{1}{2} \mathrm{~h}$ into pregnant macaques consistently failed to decrease endogenous production of progesterone. Since the half-life of progesterone in the peripheral circulation of pregnant macaques, as measured by Scholl \& Wolf (1974) after a single rapid infusion of $\left[{ }^{14} \mathrm{C}\right]$ progesterone, is approximately $4 \mathrm{~min}$ for the initial phase of disappearance and $30 \mathrm{~min}$ or less for the slower component of disappearance, any control emanating from an influence of peripheral progesterone levels should have been revealed by a $3 \frac{1}{2} \mathrm{~h}$ infusion. A $24 \mathrm{~h}$ infusion in one animal also failed to reduce endogenous progesterone production. 


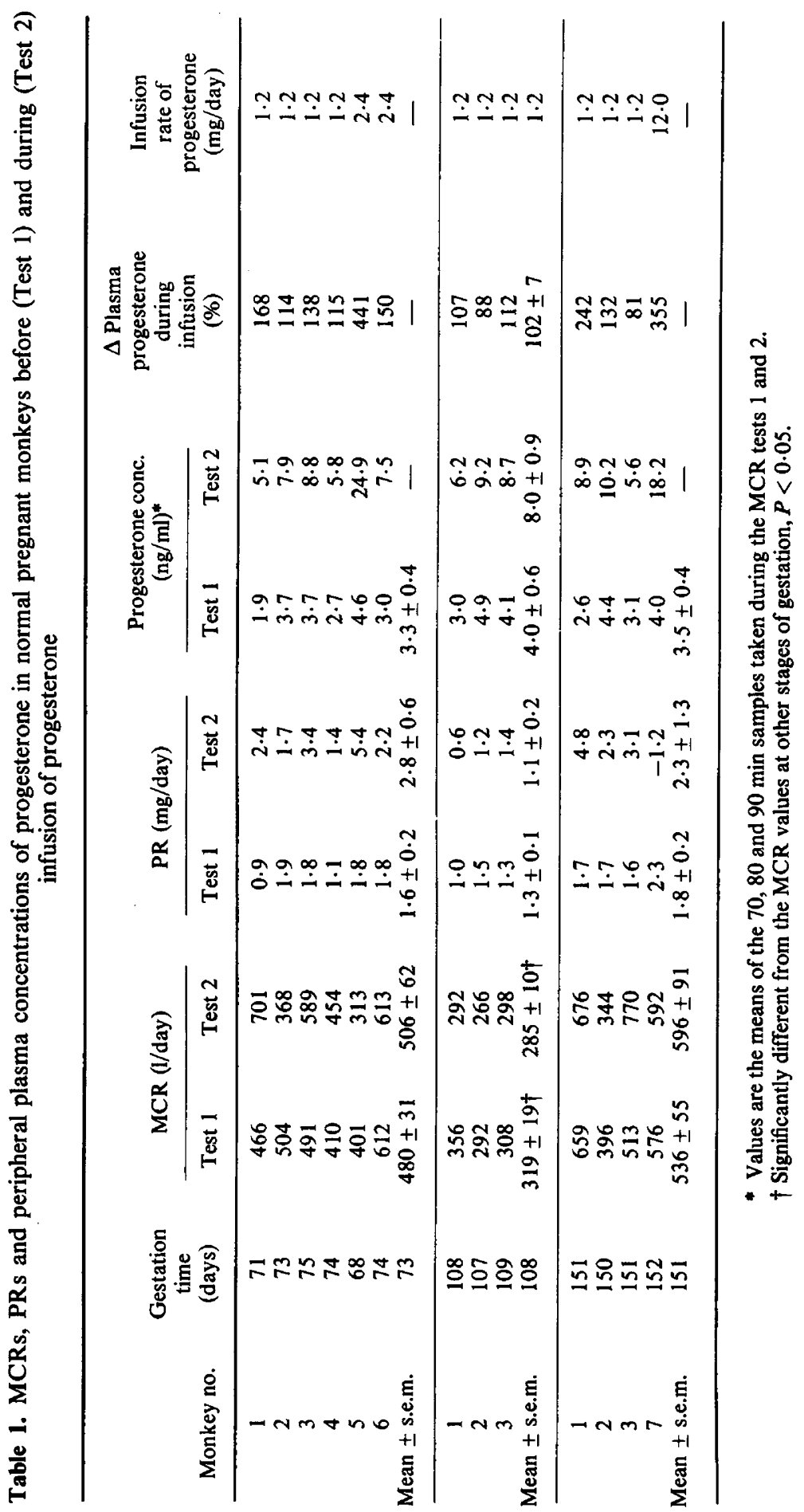




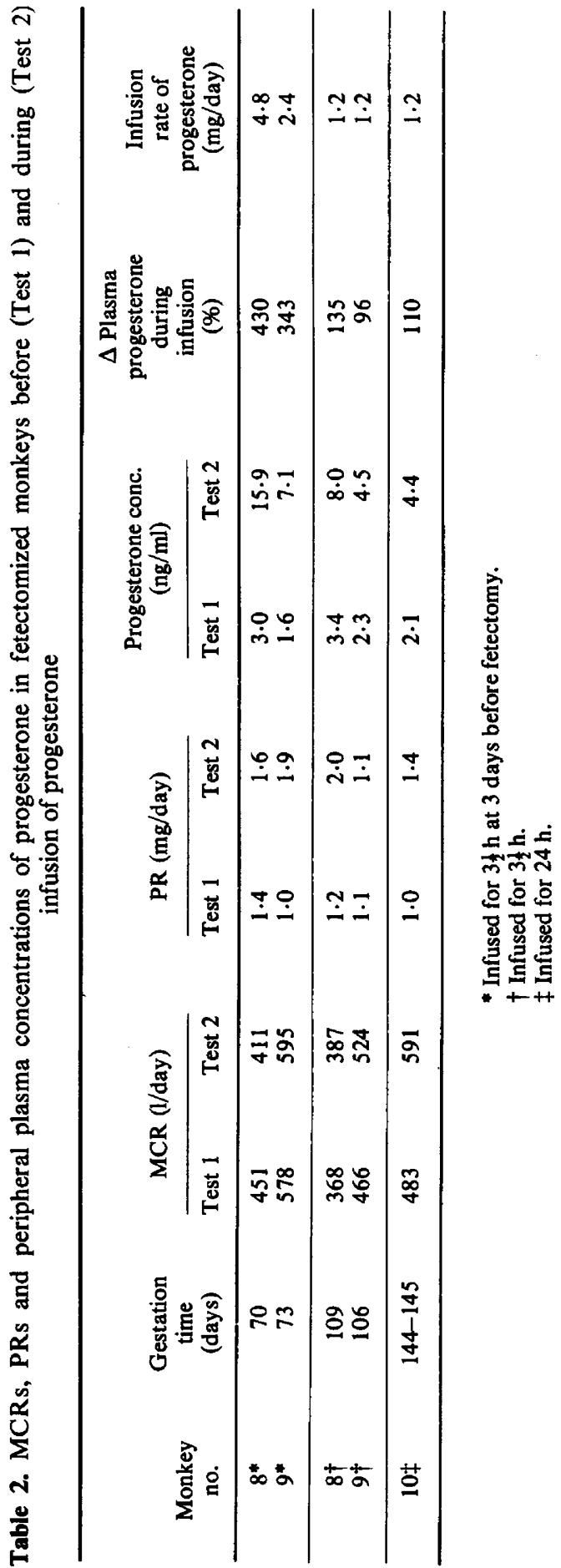


In 17 out of the 18 tests there was no reduction in the PR of progesterone although the peripheral plasma level had been more than doubled. The exception was Monkey 7 on Day 152, but on this occasion the infusion rate of non-radioactive progesterone $(12 \mathrm{mg} / \mathrm{day})$ was probably too high in comparison to the endogenous PR, because the PR actually measured during the nonradioactive progesterone infusion includes both endogenously produced (PR 2) and administered progesterone. As the latter amount becomes larger, the absolute error in the measurement of the PR increases and invalidates the calculation by difference of the endogenous PR. Therefore, the infusion rates of $12.0,4.8$ and $2.4 \mathrm{mg} / \mathrm{day}$, used at the beginning of this study, were replaced by a rate of $1.2 \mathrm{mg} /$ day.

Changes in peripheral plasma progesterone levels did not appear to affect production, but effects at sites less subject to influence by the infused progesterone (e.g. fetus, placenta or uteroovarian circulation) cannot be excluded. Infusions of progesterone sufficient to raise peripheral plasma levels to the extent shown in the present study might be expected to raise levels in the uterine veins by the same absolute amount. However, the progesterone levels in these vessels during pregnancy would be higher and the percentage increase would be smaller and the biological impact presumably less. Previous data (Thau et al., 1976a) have indicated that uterine vein progesterone levels decline during the second half of gestation in the macaque, reaching levels near term which are only about twice the peripheral plasma values. If control over progesterone production emanated from sites in the uterine vein, the most advantageous time to demonstrate such control, using our method, would be near term, when progesterone infusion would be expected to have the greatest relative effect on uterine vein concentrations. However, studies carried out near term also failed to reveal a negative feedback effect of progesterone infusion over the PR for progesterone.

Albrecht \& Townsley (1976) studied the MCR of progesterone in baboons before and after the infusion of progesterone. Although peripheral concentrations of progesterone were increased 2-fold in pregnant and 6-fold in non-pregnant animals, the MCR of progesterone was unchanged. The fact that the MCR values of fetectomized monkeys were similar to those of normally pregnant females in our study indicates that the fetus plays no direct role in the metabolism of ovarian and/or placental progesterone during pregnancy.

We thank Dr K. Sundaram for the progesterone-BSA conjugate, Ms Linda Seidman for skilful technical assistance, and the caretakers of the monkey colony.

\section{References}

Albrecht, E.D. \& Townsley, J.D. (1976) Metabolic clearance and production rates of progesterone in non-pregnant and pregnant baboons (Papio papio). Endocrinology 99, 1291-1294.

Lanman, J.T., Thau, R., Sundaram, K., Brinson, A. \& Bonk, R. (1975) Ovarian and placental origins of plasma progesterone following fetectomy in monkeys (Macaca mulatta). Endocrinology 96, 591-597.

Neill, J.D., Johansson, E.D.B. \& Knobil, E. (1969) Patterns of circulating progesterone concentrations during the fertile menstrual cycle and the remainder of gestation in the rhesus monkey. Endocrinology 84, $45-48$.

Resko, J.A. (1971) Sex steroids in adrenal effluent plasma of the ovariectomized rhesus monkey. J. clin. Endocr. Metab. 33, 940-948.
Scholl, S.A. \& Wolf, R.C. (1974) The metabolic clearance rate of progesterone in the pregnant rhesus monkey. Endocrinology 95, 1287-1292.

Tait, J.F. (1963) Review: The use of isotopic steroids for the measurement of production rates in vivo. J. clin. Endocr. Metab. 23, 1285-1297.

Thau, R.B. \& Lanman, J.T. (1975) Metabolic clearance rates (MCR) and production rates (PR) of plasma progesterone in pregnant and pseudopregnant rabbits. Endocrinology 97, 454-457.

Thau, R.B., Lanman, J.T. \& Brinson, A. (1976a) Progesterone concentrations, metabolic clearance rates and production rates in pregnant and fetectomized rhesus monkeys. Proc. 58th Ann. Meeting, Endocrine Soc., Abstr. 246, p. 179.

Thau, R.B., Lanman, J.R. \& Brinson, A. (1976b) 
Declining plasma progesterone concentration with advancing gestation in blood from umbilical and uterine veins and fetal heart in monkeys. Biol. Reprod. 14, 507-509.

Thau, R.B., Lanman, J.R. \& Brinson, A.O. (1977) Metabolic clearance rates, production rates and concentrations of progesterone in pregnant rhesus monkeys. Biol. Reprod. 16, 678-681.

Thorneycroft, I.H. \& Stone, S.C. (1972) Radioimmunoassay of serum progesterone in women receiving oral contraceptive steroids. Contraception 5, 129146.

Walsh, S.W., Wolf, R.C. \& Meyer, R.K. (1974) Progesterone, progestins and 17-hydroxypregn-4ene-3,20-dione in the utero-ovarian, uterine and peripheral blood of the pregnant rhesus monkey. Endocrinology 95, 1704-1710.

Received 17 June 1978 